\title{
CARIOTIPOS DE ESPECIES DE TURNERA (TURNERACEAE)
}

\author{
por VIVIANA G. SOLIS NEFFA ${ }^{1}$
}

\section{Summary}

The karyotypes of 6 species of Turnera are described. The karyotype of $T$. melochioides (Leiocarpae), $2 n=2 x=14$, is composed by $10 m+4 s m$. In T. grandiflora, T. candida, $T$. scabra, $T$. hermannioides and $T$. Joelii (Canaligerae), all with $2 n=2 x=10$, the karyotype formula is $8 m+2 s m$. These species may be distinguished by the mean chromosome length, the size and position of satellites and the asymmetry indices. T. scabra with autopolyploid cytotype shows shorter chromosomes and more symmetric karyotypes. Previous reports for 6 taxa are confirmed.

\section{Introducción}

El género Turnera incluye cerca de 100 especies distribuidas en 9 series (Urban, 1883) y es el más estudiado citológicamente de la familia Turneraceae. Estudios citológicos realizados en seis series (Fernández, 1987) demostraron que existen tres números básicos; el más frecuente es $x=7$, encontrándose $x=13$ en la serie monotípica Papilliferae y $\mathrm{x}=5$ en Canaligerae. La poliploidía desempeñó un papel importante en la evolución del género, hallándose niveles de ploidía desde $2 x$ hasta $10 x$ (Raman \& Kesavan, 1964; Barrett, 1978; Arbo \& Fernández, 1983; Shore \& Barrett, 1985; Fernández, 1987). Hasta el momento se conocen los números cromosómicos de 31 especies (Fernández, 1987) y los cariotipos de 16. De éstas, 4 corresponden a Salicifoliae, Leiocarpae y Papilliferae y las 12 restantes pertenecen a Canaligerae. Las especies de esta última serie muestran un cariotipo básico haploide formado por $4 \mathrm{~m}+1 \mathrm{sm}$, considerado el cariotipo fundamental (Solís Neffa y Fernández, 1993). En este trabajo se estudian cromosómicamente 8 taxones. Se describen por primera vez los

\footnotetext{
1 Becaria del CONICET. Instituto de Botánica del Nordeste, C.C.209, (3400) Corrientes, Argentina. Proyecto subsidiado por CONICET y la Secretaría de Ciencia y Técnica de la UNNE.
}

cariotipos de 5 especies diploides y de los citotipos diploide y tetraploide de T. scabra, siendo su estudio de importancia dada la gran cantidad de poliploides que presenta eì género.

\section{Material y Métodos}

El material empleado se cita en la Tabla 1. Los ejemplares testigo se encuentran depositados en el Herbario CTES. Para la obtención de los preparados de mitosis se siguió la técnica detallada en Solís Neffa \& Fernández (1993). Se analizaron 10 metafases por especie, estimándose para cada una de ellas los siguientes parámetros:

- Longitud media (LM)

- Razón promedio entre brazos $(A R)=(b / B) /$ $\mathrm{n}$

- Razón entre la longitud par más corto/par más largo (CL)

- Volumen cromosómico (VC) = long. total $\times 2$ $\times 3,14 \times(\text { ancho } / 4)^{2}$

b: longitud del brazo corto; B: longitud del brazo largo; n: número de cromosomas.

Los idiogramas fueron realizados sobre la base de las medias de las longitudes de los cromosomas y de los índices centroméricos. La nomenclatura utilizada es la propuesta por Levan et al. (1964). La estimación de la asimetría de los cariotipos se obtuvo según la razón pro- 
medio entre los brazos y también según los índices $\mathrm{A}_{1}$ y $\mathrm{A}_{2}$ de Romero Zarco (1986). El uso de estos dos índices y su representación gráfica en un diagrama de dispersión, permite realizar comparaciones cuando las diferencias de los cariotipos son muy pequeñas. El primer índice $\left(A_{1}\right)$ mide la asimetría intracromosómica y se calcula mediante la fórmula:

$$
A_{1}=\frac{1-\sum\left(\frac{b}{B}\right)}{n}
$$

Siendo b y B las longitudes promedio de los brazos cortos y largos, respectivamente, de cada par de cromosomas homólogos; n es el número de pares o grupos de cromosomas homólogos.

El segundo índice $\left(A_{2}\right)$ mide la asimetría intercromosómica y se estima usando el coeficiente de dispersión de Pearson:

$$
A_{2}=\frac{s}{\tilde{x}}
$$

s es la desviación típica y $\tilde{x}$ la media de la longitud de los cromosomas.

Los parámetros e índices estimados para las especies diploides de Canaligerae fueron comparados mediante análisis de la varianza (ANOVA) luego del test de Bartlett de homogeneidad de las varianzas. Las diferencias entre cada par de medias, fueron calculadas mediante el test de Tukey.

\section{Resultados}

En la Figura 1 se muestran las metafases mitóticas y en la Figura 2 los idiogramas de las especies analizadas.

Serie Leiocarpae. T. melochioides Cambess., $2 n=2 x=14$, posee 5 pares metacéntricos y dos pares submetacéntricos (Fig. 2). No se observa-

\begin{tabular}{|c|c|c|c|}
\hline Especie & & $2 n$ & Coleccionista y Procedencia \\
\hline \multicolumn{4}{|l|}{ Turnera } \\
\hline Serie Cat & $\begin{array}{l}\text { T. melochioides Cambess. } \\
\text { ae }\end{array}$ & 14 & Krapovickas 36653, Bolivia, Santa Cruz \\
\hline & T. grandiflora (Urb.) Arbo & $10^{*}$ & Solís Neffa et al. 42, Argentina, Corrientes. \\
\hline & T. candida Arbo & $10^{*}$ & $\begin{array}{l}\text { Hatschbach } 56662 \text { bis, Brasil, BA, cuit. } \\
\text { Corrientes. }\end{array}$ \\
\hline & « & $10^{*}$ & Hatschbach 56641, Brasil, 3A. \\
\hline & T. scabra Millsp. & 10 & Breed!'ove 47230, Mexico, Chiapas. \\
\hline & " & 20 & Barrett 1126, Venezuela, Calabozo. \\
\hline & « & 20 & $\begin{array}{l}\text { Jiménez 8769, Rep. Dominicana, prov. La } \\
\text { Vega. }\end{array}$ \\
\hline & T. subulata $\mathrm{Sm}$. & $20^{*}$ & Arbo 5451, Brasil, BA. \\
\hline & T. Krapovickasil Arbo & $10^{*}$ & $\begin{array}{l}\text { Krapovickas \& Fortunato 43922, Bolivia, } \\
\text { Santa Cruz. }\end{array}$ \\
\hline & « & $20^{*}$ & Krapovickas 40303, Argentina, Salta. \\
\hline & Thermannioides Cambess. & $10^{*}$ & Hatschbach 56826, Brasil, BA. \\
\hline & « & $10^{*}$ & Hatschbach 56828, Brasil, BA. \\
\hline & T. Joelii Arbo & $10^{*}$ & Hatschbach 44230, Drasil, BA. \\
\hline & « & 10 & Barrett \& Shore 1373, Brasil, BA. \\
\hline
\end{tabular}

Tabla 1. Especies estudiadas.

* Recuentos de nuevas accesiones. 


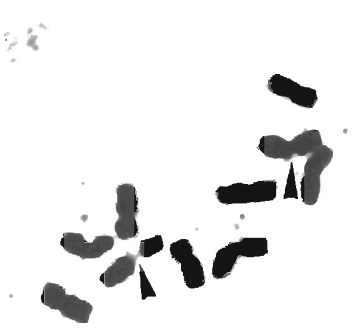

A
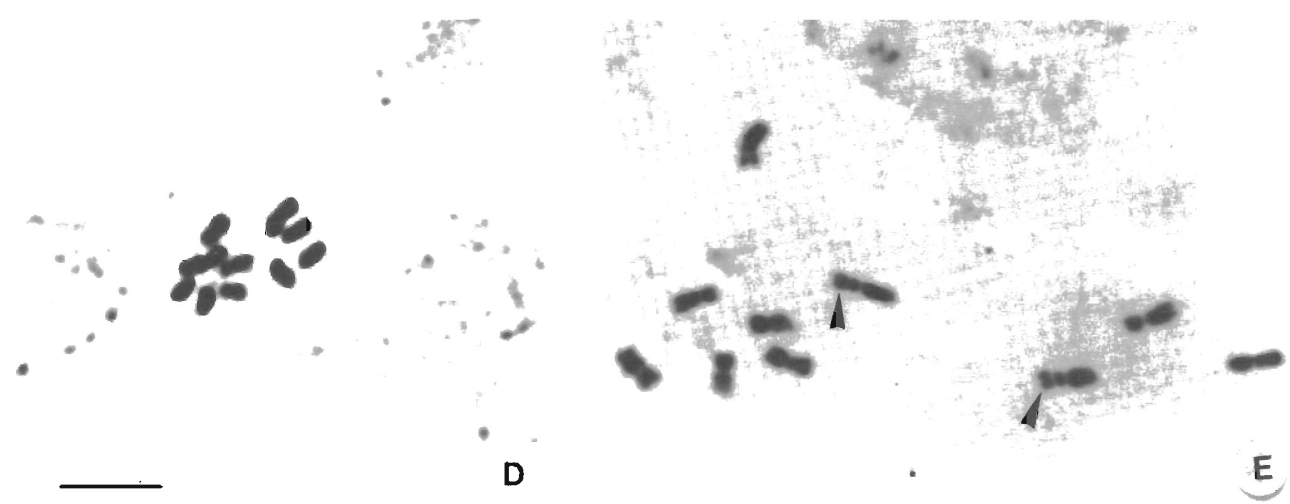

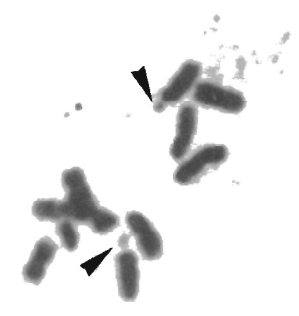

B
C

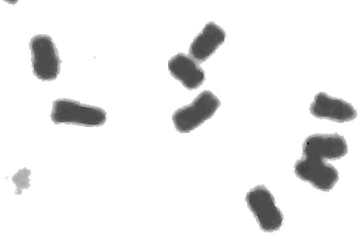

Fig. 1. Metafases mitóticas. A, T. grandiflora; B, T. candida; C, T. scabra; D, T. hermannioides; E, T. Joelii. Las flechas señalan los cromosomas con satélite. Escala $=5 \mu \mathrm{m}$.

Tabla 2. Caracterización numérica de los cariotipos de Turnera (Serie Canaligerae).

\begin{tabular}{lllllll}
\hline Especie & $\begin{array}{l}\mathrm{LM} \\
(\mu)\end{array}$ & $\mathrm{CL}$ & $\mathrm{AR}$ & $\mathrm{A}_{1}$ & $\dot{A}_{2}$ & $\begin{array}{l}\mathrm{VC} \\
\left(\mu^{3}\right)\end{array}$ \\
\hline T. grandiflora & $2.98 \mathrm{a}$ & $0.70 \mathrm{a}$ & $0.76 \mathrm{a}$ & $0.23 \mathrm{a}$ & $0.17 \mathrm{ab}$ & $9.66 \mathrm{a}$ \\
T. candida & $2.55 \mathrm{ab}$ & $0.75 \mathrm{a}$ & $0.74 \mathrm{a}$ & $0.25 \mathrm{a}$ & $019 \mathrm{a}$ & $9.31 \mathrm{a}$ \\
T. scabra & $2.53 \mathrm{ab}$ & $0.65 \mathrm{a}$ & $0.81 \mathrm{a}$ & $0.19 \mathrm{a}$ & $0.10 \mathrm{~b}$ & $8.48 \mathrm{a}$ \\
T. hermannioides & $2.25 \mathrm{~b}$ & $0.72 \mathrm{a}$ & $0.78 \mathrm{a}$ & $0.21 \mathrm{a}$ & $0.12 \mathrm{ab}$ & $7.58 \mathrm{a}$ \\
T. Joelii & $2.37 \mathrm{ab}$ & $0.69 \mathrm{a}$ & $0.78 \mathrm{a}$ & $0.21 \mathrm{a}$ & $0.11 \mathrm{ab}$ & $3.28 \mathrm{a}$ \\
F(ANOVA) & $4.21^{* *}$ & 1.09 & 0.85 & 1.41 & $4.22^{* *}$ & 3.56 \\
\hline
\end{tabular}

${ }^{* *} \mathrm{p}<.01$

Nota: Los valores de la misma columna seguidos de la misma letra no presentan diferencias significativas. Abreviaturas ver Material y Métodos

ron satélites debido, probablemente, a que los cromosomas son muy pequeños (LM=1.33 $\mu \mathrm{m})$. La razón promedio entre brazos $(\mathrm{AR}=0.74)$ y los índices de asimetría, $\mathrm{A}_{1}=0.25$ y $\mathrm{A}_{2}=0.28$, indican que es un cariotipo simétrico.
Serie Canaligerae. El cariotipo diploide de las cinco especies analızadas está compuesto por $8 \mathrm{~m}+2 \mathrm{sm}$, observándose un solo par de cromosomas con satélite, que siempre se encuentra en el brazo corto, aunque varía en 


$$
123567
$$

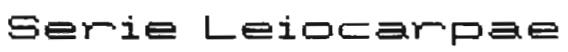

T. meløモhiøiஏes

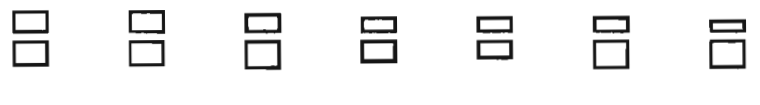

$m$

$\equiv m$

Serie దarาaligerae

T. graindiflora
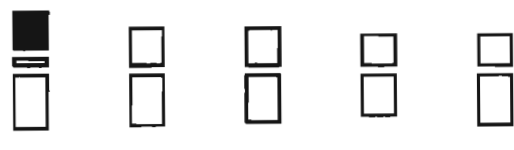

$m$

$\equiv m$

T. С®าロida

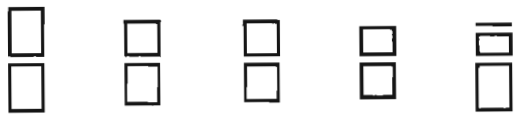

m

$\equiv m$

T. 트ดルョ

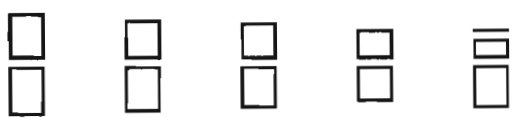

$m$

$\equiv m$

T. hermanhioides

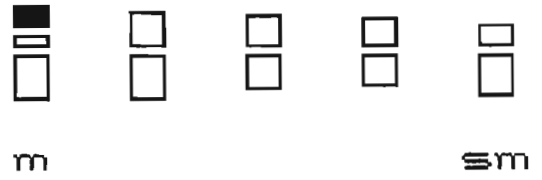

T. Jロelii

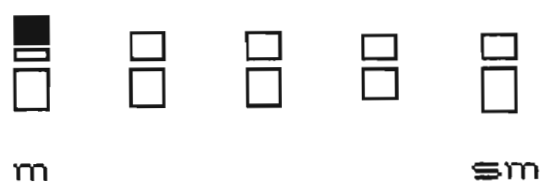

Fig. 2. Idiogramas de Turnera. Escala $=5 \mu \mathrm{m}$.

tamaño y posición según las especies. T. candida Arbo (Fig. 1B) y T. scabra Millsp. poseen microsatélite en el par submetacéntrico; T. grandiflora (Urb.) Arbo (Fig.1A), T. hermannioides Cambess. y T. Joelii Arbo (Fig. 1E) macrosatélite en el par 1. El análisis de la 
varianza entre las medias de los distintos parámetros e índices resultó ser significativo para la longitud cromosómica media y el índice de asimetría intercromosómica (Tabla 2). Las especies analizadas poseen una longitud cromosómica promedio de $2.53 \mu \mathrm{m}, T$. hermannioides ( $\mathrm{LM}=2.25 \mu \mathrm{m}$ ) posee los cromosomas más pequeños y $T$. grandiflora ( $\mathrm{I}, \mathrm{M}=2.98 \mu \mathrm{m})$ los más grandes. La razón entre brazos (AR) varía desde 0.74 en $T$. candida a 0.81 en T. scabra correspondiendo a la categoría de metacéntricos según Levan et al. (1964). Los valores de $A_{1}$ obtenidos indican que existe poca variación entre las longitudes de los brazos cromosómicos. El cociente CL y el índice $\mathrm{A}_{2}$ demuestran que la diferencia de tamaño entre los cromosomas de cada una de las especies es pequeña. T. scabra es la única especie aquí estudiada con citotipos diploide, $2 n=2 x=10$, y tetraploide, $2 n=4 x=20$. El cariotipo de este último posee $16 \mathrm{~m}+4 \mathrm{sm}$, es decir repetido cuatro veces el cariotipo básico haploide. Los cromosomas son más pequeños y el cariotipo es más simétrico.

\section{Discusión y Conclusiones}

Las especies de Tumera estudiadas muestran cariotipos simétricos y uniformes, siendo sus cromosomas pequeños. La serie Leiocarpae $(x=7)$ es la más numerosa del género ya que cuenta con 39 especies (Arbo, 1986) sin embargo, sólo se conocen los números cromosómicos de 8 de ellas (Fernández, 1987) y los cariotipos de $T$. pumilea L., $10 m+4 s m$ y de $T$. Hassleriana Urb., $12 \mathrm{~m}+2 \mathrm{sm}$ (Solís Neffa \& Fernández, 1993). Se suma ahora T. melochioides que comparte con $T$. pumilea la fómula cariotípica y el tamaño reducido de sus cromosomas.

La serie Canaligerae $(\mathrm{x}=5)$ está constituida por aproximadamente 22 especies que se reúnen en dos grupos, diferenciados entre sí por caracteres seminales (Arbo, 1986). Uno de los grupos constituye el complejo T. ulmifolia L. (Arbo, 1986), donde se conocen 7 especies diploides, tres con flores blanco-azuladas ( $T$. caerulea DC., T. grandiflora y $T$. candida) y cuatro con flores amarillas (T. Krapovickasii Arbo, $T$. concinna Arbo, T. scabra y T. subulata Sm.). EJ otro grupo comprende varias especies tropicales (Femández \& Arbo, 1.989), entre ellas T. hermannioides y $T$. Joelii, ambas de flores amarillas. Las especies de cada grupo, están relacionadas genéticamente entre sí (Aroo \& Fernández, 1987). Las 5 especies de Canaligerae aquí estudiadas, como las estudiadas en un trabajo previo (Solís Neffa \& Fernández, 1993) muestran el mismo cariotipo básico haploide formado por $4 \mathrm{~m}+1 \mathrm{sm}$. No obstante, estos taxones se pueden diferenciar por el tipo y posición de los satélites, por el tamaño relativo de los cromosomas dentro de cada uno de los cariotipos (índice $\mathrm{A}_{2}$ ) y por la longitud cromosómica media (LM). En la Fig. 2 se observa que los cariotipos de $T$. hermannioides y $T$. Joelii, que no pertenecen al complejo T. ulmifolia (Arbo \& Fernández, 1987), son semejantes al de $T$. grandiflora. Estas especies poseen macrosatélite en el par 1 , sin embargo, éste es de menor tamaño en $T$. hermannioides. $T$. grandiflora y $T$. joelii se diferencian por la longitud cromosómica media y en el índice $\mathrm{A}_{2} . T$. candida y $T$. scabra también presentan similitudes en sus carrotipos pero difieren en los valores del índice de asimetría intercromosómica (Tabla 2).

Turnera grandiflora presenta citotipos diploide $(2 n=2 x=10)$ y octoploide $(2 n=8 x=40)$. Solís Neffa \& Fermández (1993) analizaron una accesión octoploide proveniente del Chaco paraguayo y encontraron que en su cariotipo está representado 8 veces el cariotipo fundamental. Fernández (1987) analizó la meiosis de una accesión proveniente de Mato Grosso observando que citológicamente se comporta como autooctoploide. El cariotipo del citotipo diploide estudiado aquí presenta cromosomas de mayor longitud media y diferencias respecto al tamaño y posición del satélite en reiación al octoploide. El diploide posee macrosatélite en el brazo corto dei par 1 y el octoploide microsatélite en el brazo corto del par 3 (Solís Neffa \& Fernández, 1993). Lo expuesto sugiere la necesidad de analizar más accesiones de ambos citotipos.

Entre las especies de flores amarillas, los datos morfológicos y citológicos (Fernández \& Arbo, 1989) indican que T. subulata y T. scabra son especies muy afines. Los cariotipos confirman estos datos, ambas poseen microsatélite en el par submetacéntrico y los cariotipos más simétricos entre las especies estudiadas (Fig. 3). 


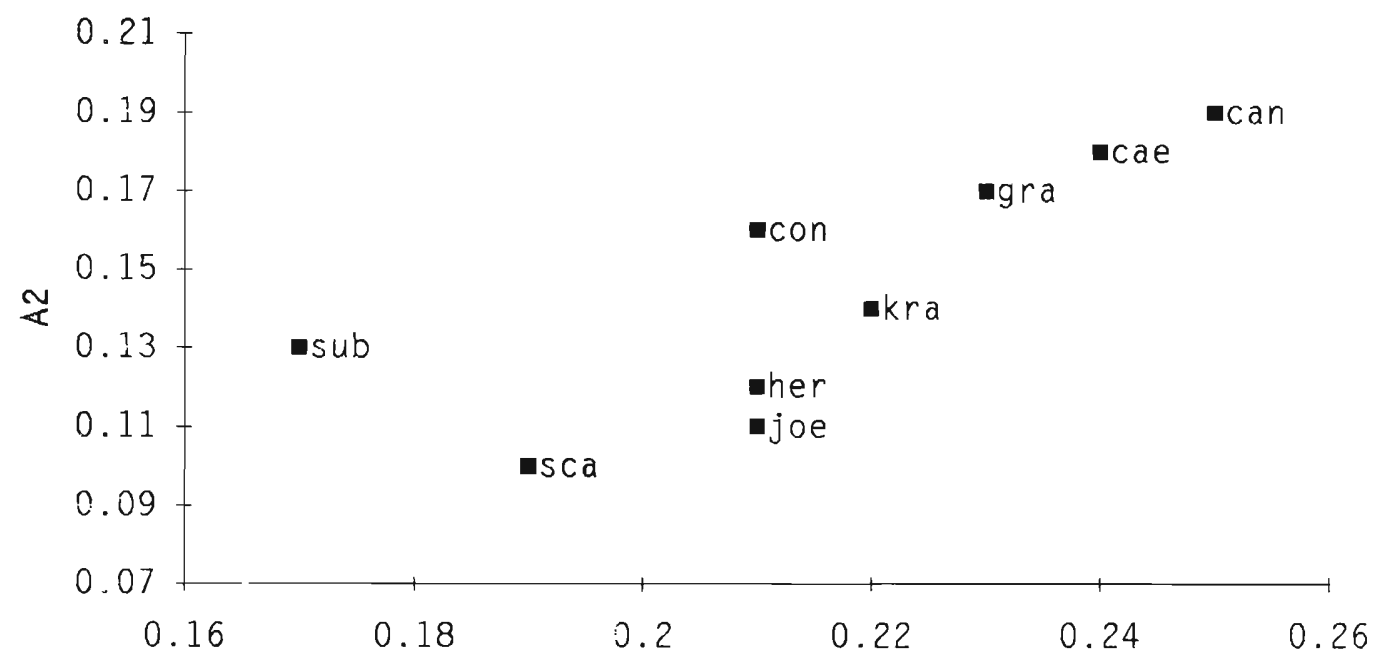

A1

Fig. 3. Diagrama de dispersión. cae, Turnera caerulea; can, T. candida; con, T. concinna; gra, T. grandiflora; her, T. hermannioides; joe, T. Joelii; kra, T. Krapovickasii; sca, T. scabra; sub, T. subulata. Los datos de T. caerulea, T. concinna, T. Krapovickasii y $T$ subulata fueron tomados de Solís Neffa \& Fernández (1993).

Turnera subulata, T. Krapovickasii y T. scabra presentan también citotipos $4 x$. Solís Neffa \& Fernández (1993) analizaron los citotipos diploide y autotetraploide de las dos primeras especies y observaron que en los diploides los cromosomas son de mayor longitud pero de menor simetría, conservándose el tipo y posición de los satélites. Los cariotipos de los citotipos $2 x$ y $4 x$ de $T$. scabra confirman lo observado por dichos autores y está de acuerdo con el criterio de Stebbins (1938) de que la poliploidía está correlacionada con una disminucion de la longitud cromosómica.

El diagrama de dispersión (Fig. 3) permite realizar comparaciones de variaciones numericas continuas y detectar cambios pequeños y paulatinos en la evolución de los cariotipos. En él se observa que las especies de flores blancoazuladas poseen ios cariotipos más asimétricos, ockipando la posición más extrema. $T$. hermannioides y $T$. joelii se sitúan en la posición central próximas a T. concinna y T. Krapovickasii de acuerdo a los valores de $\mathrm{A}_{1}$, pero difieren de estas últimas por los valores de $\mathrm{A}_{2}$.

\section{Agradecimientos}

Deseo expresar mi agradecimiento al Est. Eduardo Porcel y a la Ing. Agr. Silvia Mazza de
Gaiad por su asesoramiento en el análisis estadístico de los datos.

\section{Bibliografía}

ARBO, M. M. 1986. Paraguay, centro importante de especiación en las Turneráceas. Candollea 41: 212218.

ARBO, M. M. \& A. FERNANDEZ. 1983. Posición taxonómica, citología y palinología de tres niveles de ploidía de Turnera subulata Smith. Bonplandia 5 (23): 211-226

- 1987. Cruzamientos intra e interespecíficos en Turnera, Serie Canaligerae. Bonplandia 6 (1): 23-38.

BARRETT, S. C. H. 1978. Heterostyly in a tropical weed: the reproductive biology of the Turnera ulmifolia complex (Tumeraceae). Canad. J. Bot. 56 (15): 1713-1725.

FERNANDEZ, A. 1987. Estudios cromosómicos en Turnera y Piriqueta (Turneraceae). Bonplandia 6 (1):1-21.

FERNANDEZ, A. \& M. M. ARBO. 1989. Relaciones genómicas entre cuatro especies diploides de Turnera con flores amarillas (Serie Canaligerae). Bonplandia 6 (2): 93-109.

LEVAN, A., K. FREDGA \& A. A. SANDBERG. 1964. Nomenclature for centromeric position on chromosomes. Hereditas 52 (2): 201-220.

RAMAN, V. S. \& P. C. KESAVAN. 1964. Meiosis and nature of polyploidy in Turnera ulmifolia. J. Indian Bot. Soc. 43 (3): 495-497.

ROMERO ZARCO, C. 1986. A new method for estimating karyotype asymmetry. Taxon 35 (3): 526-530.

SHORE, J. \& S. C. H. BARRET. 1985. The genetics of distyly 
and homostyly in T. ulmifolia L. (T'umeraceae). Heredity 55: $167-174$.

SOLIS NEFFA, V \& A. FERNANDEZ. 1993. Estudios cromosómicos en especies de Turnera (Turneraceae). Bonplandia 7 (1-4): 101-118.
STEBBINS, G. L. 1938. Cytological characteristics associated with the different growth habits in the dicotyledons. Amer. J. Bot. 25 (3): 189-198.

URBAN, I. 1883. Monographie der familie der Turneraceen. Jahrb. Königl. Bot. Gart. Berlin 2: 1-152. 